\title{
Preschoolers' Metaphor Comprehension. Methodological Issues in Experimental Pragmatics
}

\author{
Anna BABARCZY \\ Budapest University of Technology and Economics, Hungarian Academy of Sciences \\ (Budapest, Hungary) \\ Department of Cognitive Studies, Research Institute for Linguistics \\ babarczy@cogsci.bme.hu \\ Andrea BALÁZS \\ Budapest University of Technology and Economics (Budapest, Hungary) \\ Department of Cognitive Studies \\ futrago@gmail.com

\section{Fruzsina KRIZSAI} \\ Budapest University of Technology and Economics (Budapest, Hungary) \\ Department of Cognitive Studies \\ f.krizsai@gmail.com
}

\begin{abstract}
There exists a variety of theoretical frameworks attempting to account for the nature, comprehension, and use of everyday metaphor. Since these frameworks use different operational definitions of metaphor, they tend to view the psycholinguistic process of comprehending metaphorical language and the various factors that may play a role in metaphor processing from different perspectives. The first part of the paper briefly summarizes four of these theoretical approaches to everyday metaphor (Conceptual Metaphor Theory, Similarity Theory, Relevance Theory, and the Optimal Innovation Hypothesis) and discusses some consequences of the diversity of theories that present a puzzle or prove to be undesirable for empirical research. The areas discussed include the various dimensions of metaphor categorization, the role of linguistic context, and the effects of linguistic and non-linguistic cognitive skills of the comprehender. Drawing on the discussion in the first part, the second part of the paper outlines an experiment designed with reference to Giora's Optimal Innovation Hypothesis in which preschoolers' metaphor comprehension is explored as a function of the familiarity of the expression's literal meaning and the perceived creativity of the metaphorical use. This experiment further explores the relationship between children's metaphor comprehension and
\end{abstract}


other cognitive abilities such as intention attribution. This method allows us to quantify metaphor comprehension and preference in the context of pragmatic development and general cognitive skills.

Keywords: metaphor theories, experimental pragmatics, methodology, cognitive abilities

\section{Introduction}

Even though a large number of studies have been carried out on the development of metaphor comprehension among children, it is difficult to obtain an overall picture of the process because the different studies rely on a wide range of mutually exclusive theoretical perspectives. While Lakoff and Johnson's (1980) Conceptual Metaphor Theory was a turning point in cognitive linguistics, there are other approaches to metaphor that provide a framework for research on the development of metaphor comprehension and use, such as Similarity Theory, Relevance Theory, and the Optimal Innovation Hypothesis. Based on these theories, a metaphor may be regarded as a result of conceptual mapping or of similarity-based analogical categorization or else an instance of innovative language use, and metaphor understanding may be a basic ability underlying human conceptualization or a special component of pragmatic competence or perhaps a consequence of the human ability to interpret messages as optimally relevant. The problem is further complicated by the fact that some theories, such as Conceptual Metaphor Theory, are little concerned with issues regarding metaphor in language use and have nothing to say about pragmatic competence, while pragmatic approaches to the question tend not to explicate their theoretical background in any detail (cf. Lorusso 2007, Pinto et al. 2008). In this paper, we shall first briefly summarize the main principles of Conceptual Metaphor Theory, Similarity Theory, Relevance Theory, and the Optimal Innovation Hypothesis. Next, we shall discuss some practical issues concerning metaphorical language comprehension, including different categories of metaphor, the linguistic context of metaphorical expressions, and the roles of linguistic and non-linguistic cognitive abilities, and their relation to the above theoretical frameworks. Finally, we outline an experimental design that fruitfully combines the different features of the approaches discussed. 


\section{Conceptual Metaphor Theory, Similarity Theory, Relevance Theory, and the Optimal Innovation Hypothesis with some critical remarks}

Lakoff and Johnson's (1980) metaphor theory is a fundamental work in cognitive linguistics: it focuses on the conceptual nature of the phenomenon, which allows metaphor to be regarded as part of everyday communication and thought. Conceptual Metaphor Theory (hereafter CMT) firmly embeds language in thought processes: "metaphor is pervasive in everyday life, not just in language but in thought and action" (1980: 3). It argues for a metaphorical conceptual system underlying linguistic expressions.

In this model, metaphor is the result of a systematic mapping process between conceptual and linguistic structures, where the conceptual structures emerge from an association between two conceptual domains: an abstract target domain, which is beyond direct perceptual experience, and a relatively concrete source domain, which is easily accessible.

(1) I send her my love.

(2) I am falling in love with you.

The abstract concept of LOVE is structured similarly to a physical entity in examples (1) and (2): love in (1) is based on the mapping of LOVE and PHYSICAL овјест. Both are viewed as being located in the physical space; they can be moved from one place to another. Example (2) also represents LOvE as an object, but here it is a CONTAINER into which an entity may fall. Although both examples make the target domain of LOVE accessible, the sources differ. These expressions highlight different attributes of the source domain and the target domain (cf. the term "main meaning focus" in Kövecses 2010).

Metaphorical mapping and the interpretation of a whole expression (the result of the mapping) are context-dependent. Metaphor comprehension requires context (1980: 12): metaphorical expressions may have widely differing interpretations depending on the current discourse situation. This implies that the process of interpreting metaphorical expressions is very similar to the process of comprehending literal language in this model: they both have a schematic lexical and an actual meaning. While there is a schematic and conventional way of mapping, the actual meaning is only possible to be interpreted successfully in a physical, situational, and linguistic context.

Lakoff and Johnson (1980: 19) claim that it is difficult to distinguish the physical from the cultural basis of metaphor because cultural conventions influence the choice of a physical perspective. However, the authors make only some 
speculative claims about the direct experiential basis of metaphors - reflecting on their own introspective approach. Though CMT itself raises certain questions, these are not crucial from the introspective viewpoint of the original theory. They nevertheless have an important role in empirical metaphor research, and that is why the current study focuses on the issues of context, language experience, and the cognitive and cultural embeddedness of metaphors. These topics are cardinal in metaphor comprehension, including its development in the child, while they also bear relevance to pragmatic theory.

An earlier but still influential theory is based on Gardner and Winner's developmental perspective called Similarity Theory which regards metaphor as a result of similarity-based analogical processes (Gardner et al. 1975; Gardner and Winner 1978; Winner 1979). The authors argue that meaning overextension and pretend play are early signs of metaphorical thinking at an age when children are not yet able to understand or use metaphors. "[...W]hile young children may not be capable of producing the kinds of metaphors produced by adults, they seem to process at least a rudimentary metaphoric ability" (Winner 1979: 471). In this approach, metaphor understanding depends on the mental process of categorization, analogical class inclusion, and it is not only a question of linguistic or conceptual knowledge but perceptual experiences may also count. Gardner et al. (1975) find that metaphorical matching (e.g. pairing stimuli from different sensory modalities) develops earlier than the recognition and acceptance of dual (literal and metaphoric) meaning of a linguistic term.

Özcaliskan (2005) criticizes Similarity Theory from the perspective of CMT, arguing that it cannot account for the systematicity of metaphorical concepts nor the direction of metaphorical mapping from concrete to abstract. Furthermore, an arbitrary selection of these expressions measures not so much general metaphor comprehension but knowledge of individual items, which is more of a question of language experience. The author admits, however, that CMT, on the other hand, makes no predictions concerning metaphor comprehension and identification since it cannot distinguish between embodied metaphor, i.e. metaphor directly derived from physical experiences, and the cultural and conventional basis of metaphor use. Beaty and Silvia (2013) mention the absence of an empirical basis in CMT even though the theory assumes a hierarchical system of metaphors based on mental and direct perceptual accessibility.

In a Relevance Theoretical framework, metaphor is an instance of vague language use, which is based on logical resemblances guided by principles of relevance. This approach treats metaphor as an interpretational process: when a thought is too complex to be represented literally for some reason, the speaker may choose an expression which is not literally true but has logical and contextual implications (Sperber and Wilson 1996: 233-234). The listener will interpret this expression according to the principle of relevance, i.e. minimizing 
processing effort and maximizing cognitive effect. Wilson (2011) writes that "relevance theorists have argued that understanding utterances involves special-purpose inferential procedures that apply only in the communicative domain" (Wilson 2011: 16).

Inspired by Relevance Theory, Giora (1997, Giora et al. 2004) developed an alternative called the Optimal Innovation Hypothesis. Giora contends that listeners strive to access optimally innovative stimuli. To be optimally innovative, a linguistic stimulus should contain salient, familiar linguistic items and concepts while at the same time inducing a novel interpretation. According to Giora, a high degree of salience reduces processing costs, while the novel interpretation gives "pleasure". Optimal innovation means a "novel - less or nonsalient response to a given stimulus, which differs not only quantitatively but primarily qualitatively from the salient response(s) associated with this stimulus" (Giora et al. 2004: 116).

In recent years, hybrid approaches combining the above main theories have emerged. Tendahl (2009) argues that all of the above theories offer useful insights into the relationship between thought and metaphorical language, but he offers a model in which the main principles of cognitive linguistics and Relevance Theory are combined. (For a more detailed overview of the evolution of metaphor theories, see Stöver 2010.) In a recent contribution to the debate, Wilson (2011) summarizes the main similarities and differences between the cognitive linguistic and the relevance theoretical approaches to metaphor. She highlights the similarity of their ideas on conventionalized metaphor use, ${ }^{1}$ the role of conventionalized metaphors in the interpretation of novel ones, and the principal disagreement in the place of metaphor in human cognition: "More generally, relevance theorists see metaphors as arising primarily in linguistic communication, whereas cognitive linguists see them as arising primarily in thought" (Wilson 2011: 15). She proposes not to treat cross-domain mappings as either an exclusively thought process or an exclusively linguistic process but to adopt a more flexible approach to the question. Wilson further notes that some disagreements between the two theoretical approaches can only be settled based on new empirical results from developmental and neuropsychological studies.

This short overview of theoretical possibilities and their interrelations shows that navigation among theories is not self-evident or clear-cut because superficial similarities may hide very different underlying principles. Criticisms and discussions of the above theories also reveal that some issues are best investigated from an empirical viewpoint.

1 "[...M]any of the examples used in both relevance theory and cognitive linguistics contain metaphorical expressions whose interpretation is more or less a matter of routine" (Wilson 2011: 14). 


\section{Experimental approaches}

In the following sections, a variety of experimental approaches are discussed and compared in an effort to identify the most important theory-independent questions of the field and to show that some of the contradictions in the results are mainly caused by the use of different theoretical backgrounds as starting points. These issues include principles of metaphor categorization and the effects of context, certain verbal and nonverbal cognitive abilities, and verbal and chronological age as detailed below.

\subsection{Categorization}

Upon a review of the literature, we cannot avoid noticing that even recent experimental studies use a variety of different dimensions of metaphor categorization (e.g. verbal versus visual, sensory versus physico-psychological, creative versus conventional, novel versus familiar, etc.). It is not quite clear whether these categorizations may be interpreted as practical solutions to certain theoretical difficulties, or they are rooted in their own solid (but implicit) theoretical background.

Some experimental approaches to metaphor treat it as a special type or subcategory of figurative language. In these studies, we can detect the effects of Similarity Theory. In this framework, Melogno, Pinto, and DiFilippo (2017) distinguish sensory and physico-psychological metaphors based on the perceptual process underlying metaphorical mapping. Sensory metaphors (e.g. her hair is a bush) have a perceptual basis, such as shape or texture, while physicopsychological metaphors (e.g. he is my rock) are based on more abstract features of the source and the target domains. This method of categorization is similar to CMT's distinction between structural and orientational metaphors in that the categories are distinguished by the transparency of the mapping structure, but it differs in how transparency is defined. While CMT separates its categories by the attributes of the mapping structure, Melogno, Pinto, and DiFilippo create their classification based on the relationship between the conceptual domains.

In Melogno, Pinto, and DiFilippo's work, the distinction between verbal and visual/pictorial metaphor is based on practical considerations: although CMT emphasizes the conceptual nature of metaphors, certain experimental studies draw a sharp boundary between verbal and visual processes of metaphor comprehension and therefore work with the separate mental processes of perception and language processing. Consequently, they use unequivocally verbal metaphors as stimuli and test their comprehension using purely verbal tasks: children have to describe the meaning of each expression. 
Pouscoulous (2011), however, postulates that all metaphors require visualization or at least visual recall, and it is therefore impossible to separate verbal and visual aspects of metaphor processing. This claim relies on both empirical and theoretical considerations. Psycholinguistic and neuroimaging studies and some theoretical models (e.g. Gibbs and Matlock 2008) suggest that the understanding of ordinary language may involve the mental simulation of the meaning of what is being heard or read. This way, hearing and reading sentences with a visual semantic component involves the activation of visual brain areas and thus interferes with visual processing. This activation and interference can also be observed even when one processes a metaphorical expression describing an event impossible in its literal sense in the real world (e.g. Gibbs and Matlock 2008, Camp 2009).

The metaphor comprehension subtest of an Italian pragmatic competence test, Abilità Pragmatiche nel Linguaggio Medea (APL Medea), developed by Lorusso (2007) contains verbal and pictorial metaphors and asks the meanings by direct questions. This way, APL Medea is a purely verbal task using the categories of verbal and pictorial metaphors. Applied categories of these presented works show that not only the distinction but also the underlying practical and methodological considerations are important.

Another dimension of metaphor classification is cognitive and cultural salience. From the point of view of ease of comprehension and accessibility, Beaty and Silvia (2013) differentiate between creative and conventional metaphors: conventional metaphors are familiar and have a straightforward relationship between the target and the source (3a), while creative metaphors are novel, unique, tend to describe an emotional experience, and usually have an aesthetic goal (3b). With a scalar approach, the terms "creative" and "conventional" are the two extremes of linguistic variation and cultural embedment.

(3a) Music heals me.

(3b) A: Think of the most boring high-school or college class that you've ever had. What was it like to sit through?

B: It was like watching paint dry.

(Beaty and Silvia 2013)

A similar distinction, that between novel and familiar metaphors, plays a fundamental role in Giora's Optimal Innovation Hypothesis (Giora 2002, Giora et al. 2004). Mashal and Kasirer (2011) and Zheng et al. (2015) adapt the categories of Giora's original - and already experimentally tested - Optimal Innovation Theory to children's metaphor comprehension. The authors compare the results of children with Autism Spectrum Disorder (ASD) to those of typically developing children: they find that novel metaphors are easier to understand than familiar ones among the ASD group, while the opposite pattern appears among typically developing children. 
Given the definition of optimal innovation, categorizing metaphors into novel and familiar is far from being a self-evident question either when adult language users are concerned (cf. Kasirer and Mashal 2014) or when looking at the process of language development. To resolve this problem, Zheng et al. (2015) pilot tested metaphors in preparation for their experiment: they asked 10 children aged 6-7 to rate the familiarity of 25 metaphorical and 25 metonymic stories on a 7-point scale, and then they selected 14 of the highest and 14 of the lowest familiarity scores. Carriedo et al. (2016) carried out a norming study to obtain a clearer picture of familiarity, comprehensibility, and aptness, and Mashal and Kasirer (2011) relied on previous pilot experiments for metaphor selection.

\subsection{Context}

CMT and RT claim that metaphor comprehension is a strongly context-dependent process, but not all experiments follow or agree with this theoretical tenet. Carriedo et al. (2016) choose metaphorical expressions which can be understood in the absence of context. The authors argue that context helps the comprehender to distinguish relevant from irrelevant information, and the comprehension of metaphors in the absence of context is a more complex analogical categorization process in which different cognitive resources are involved. The authors therefore separate general meaning comprehension from contextual understanding. This approach treats metaphors in a very similar way to everyday literal language expressions since it is not the operation of metaphorical mapping that requires context but the context-dependent understanding of a schematic, general meaning. Pouscoulous et al. (2011), by contrast, claim that decontextualized or poorly contextualized metaphors are nothing like the natural, everyday occurrence of the phenomenon, and the meaning of a metaphorical expression cannot be deduced with any certainty in the absence of context (cf. Lakoff and Johnson 1980).

"In order to enhance metaphoric understanding, on the one hand, and encourage suppression of meaningless interpretations, on the other hand", Mashal and Kasirer (2011: 2049) embed each metaphorical expression in a sentence. Zheng et al. (2015) test metaphors in highly structured, standardized short stories, where all contexts follow the same pattern. Özcaliskan (2005) also uses a rich context even whole stories - to embed metaphorical expressions.

In their case study, Melogno, Pinto, and Orsolini (2017) use a standardized Italian instrument, Junior Metaphor Comprehension Test (Pinto et al. 2008), in metaphor comprehension assessment. The test includes some metaphors in short sentences and others embedded in short stories, i.e. the above methods are combined. Although the authors did not originally choose this design to compare the effects of different levels of context, their task could be used for that purpose. 
One problem with the use of context in computing non-literal meaning is that it is not self-evident what context should be used and in what way. In fact, Relevance Theory and the Optimal Innovation Hypothesis emphasize the role of "mindreading" as a special-purpose inferential ability to understand the communicator's intentions when uttering a metaphorical utterance. However, most of the works discussed above choose not to pay attention to the correlation between metaphor understanding and "mindreading" ability (for a few exceptions, see Kalandadze et al. 2018). In a nutshell, we can say that the identification of speaker intentions is relevant from all of the introduced theoretical perspectives: it relates to context in CMT, it is a requisite ability to understand relevant meaning in RT and an inducer of categorization in ST, but it rarely appears in explicit tests. In experimental approaches, it functions as an underlying principle which influences the role attributed to context and methodological considerations.

\subsection{Shifting, inhibition, verbal and nonverbal intelligence}

Depending on the metaphor approach and classification system used, the above mentioned experimental studies find that the success of metaphor comprehension correlates with one or another cognitive ability. The theoretical backgrounds described above make predictions clear as to the skills required (e.g.: CMT states that metaphor comprehension is part of a general-purpose cognitive and linguistic ability and is based on general conceptual knowledge, Similarity Theory assigns a role to analogical reasoning, and Relevance Theory emphasizes the role of mindreading ability). Current experimental approaches argue that all of the above are in some way dependent on cognitive control processes, specifically the ability to hold linguistic and contextual information in memory and organize it, shift attention between the different (literal and metaphorical) interpretations of a metaphorical expression, and inhibit the irrelevant interpretation. Indeed, several studies have found a correlation between cognitive control abilities and metaphor comprehension in children. Carriedo et al. (2016), for instance, look at memory organization and inhibition and find that they are good predictors of metaphor comprehension, especially in the case of children with relatively poor lexical knowledge. Mashal (2013) emphasizes the role of working memory and inhibitory control in metaphor comprehension as the foundation stones of cognitive skills such as reasoning, learning new information, and language comprehension.

In their meta-analysis, Kalandadze et al. (2018) show that language ability is a crucial factor in metaphor comprehension, and studies of the typically developing population also measure verbal intelligence. Beaty and Silvia's (2013) crystallized intelligence test, for instance, includes vocabulary and general knowledge tasks, and the results correlate with conventional metaphor comprehension but not 
with creative metaphor comprehension. Deckert et al. (2018) use the verbal subscale of the Wechsler Intelligence Scale, a subtest of receptive grammar, and a subtest of semantically inconsistent sentence correction of the German Heidelberg Evaluation of Language Development (HELD). Their analysis shows that verbal intelligence and language competence are good predictors of metaphor comprehension at the age of 9-10 years.

To conclude, it should be noted that the studies rely on different theoretical backgrounds and use different methods, and therefore their results cannot be automatically compared. Even within one general approach, such as the one looking at cognitive ability, different studies use a variety of different tasks.

\subsection{Age}

Different experimental designs offer a heterogeneous set of results regarding metaphor comprehension in different age-groups. Pouscoulous (2011) argues that there is no a priori reason to assume that young children cannot understand metaphors as soon as they understand object properties well enough to make the relevant inferences. This claim is based on the assumption that the understanding of metaphors involves the understanding of the analogy between the actual concrete object and the abstract metaphorical one, i.e. the mapping of properties from one object onto another. Relying on these abilities, children as young as 18 months can understand that a sponge can be a rain cloud in the context of pretend play. Although young communicators may easily participate in pretend play, their ability to understand metaphors is hampered by their lack of world knowledge as well as the underdevelopment of language skills and of some cognitive abilities such as working memory and cognitive control.

While Pouscoulous finds evidence for metaphor production and comprehension before the age of 3, standardized metaphor comprehension tests (Lorusso 2007, Pinto et al. 2008) recommend starting with 4-year-old children, and Deckert et al. (2018) and Carriedo et al. (2016) measure metaphor comprehension much later, from middle childhood and early adolescence. Özcaliskan (2005) finds that 4-year-old preschoolers are already able to recognize perceptual similarity and build analogical links between source and target domains, but the active use of metaphors (e.g. in a rephrase or meaning choice task) is still under development at the age of 7-9.

According to Kalandadze et al. (2018), we should take into account the question of chronological versus mental age. The authors analyse the reliability of the extensive research on metaphor comprehension among children with Autism Spectrum Disorder compared to typically developing controls. Their meta-analysis shows, inter alia, that language abilities may be better predictors of metaphor comprehension than chronological age - and this is certainly so in 
ASD studies. The authors conclude that figurative language comprehension is closely related to core language abilities in the case of individuals with ASD, but they also point out that the question of the relationship between metaphor comprehension and core language ability and non-linguistic cognitive ability is a lot more complex than a simple correlation with chronological age.

\section{A possible experimental design}

As it is discussed above, many decisions need to be made in order to design an appropriate experiment. Since we are interested in reconciling the different approaches in order to obtain a theory-neutral picture of the development of metaphor comprehension, we have developed an experiment that combines several of the above features without sacrificing interpretability.

\subsection{Test of metaphor familiarity and pleasure}

Based on Giora's Optimal Innovation Hypothesis, we have tested 3- to 6-yearold children's comprehension of and preference for metaphorical expressions. First, we have chosen a set of 133 phrases from stories written for an audience of that age range to ensure that the expressions are appropriate for the children. The set of phrases were independently marked by three annotators specifying whether they thought each expression was metaphorical, idiomatic, or literal. The judgements were then discussed, and 54 expressions were selected that all three annotators agreed were metaphorical. The next step is to categorize the metaphors into novel versus familiar or creative versus conventional. Two methods are used for categorization: 1) the frequency of occurrence of each expression is obtained using the World Wide Web and 2) a survey is created for parents with children of the target age range. The survey asks parents to rate each expression (embedded in a short sentence) on a scale of 1 to 3 , where 1 means that their child is definitely not familiar with the expression, 2 means that their child might be familiar with the expression, and 3 means that their child is definitely familiar with the expression. The raw frequency obtained using the first method gives us an overall frequency score for each metaphor, and the results of the second method are averaged across parents to give us an age-specific familiarity score for each metaphor.

Of the 54 metaphors, 10 familiar and 10 novel ones are chosen for the familiarity and the preference tasks. In the familiarity task, the metaphors are embedded in short stories related by the experimenter. The story context is compatible with both the metaphorical and the literal interpretation of the expression (see Figure 1). 
Context: The boy lost his favourite ship. He was looking for it, but he could not find it anywhere.

Test sentence: He was so sad, he cried a river.
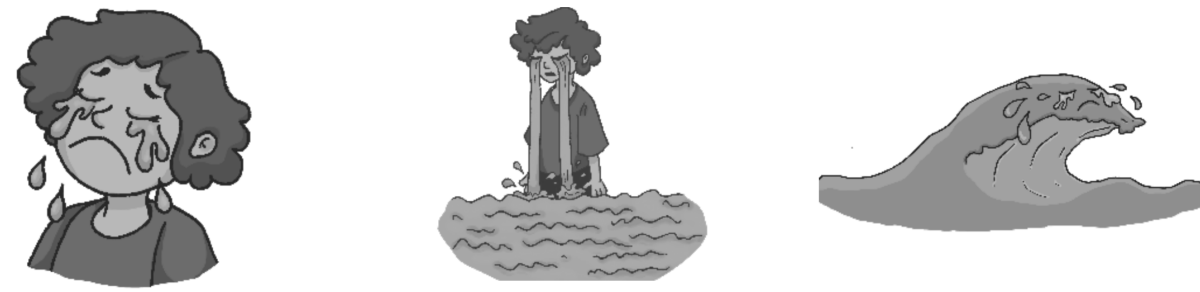

1

2

3

Figure 1. Familiarity test

After hearing the story, participants are shown three images in random order: 1) a picture depicting the metaphorical meaning of the test expression, 2) a picture depicting the literal meaning, and 3) an unrelated picture with similar visual attributes to the other two. The task is to select the picture that best matches the story.

In the preference task (see Figure 2), each of three bunny toys describe a picture in a single sentence in one of the three different ways. One of the bunnies uses a metaphorical expression (c) in the description, while the other two bunnies describe the picture using only literal language (a and b). All three sentences are accurate descriptions of the image. The children's task is to choose the description they like best.
a) He was so sad, he cried a lot.
b) He was so sad, his tears were falling.
c) He was so sad, he cried a river in his sadness.

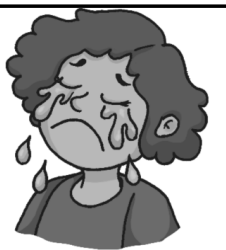

Figure 2. Pleasure test

\subsection{Intention attribution}

As CMT, RT, and several experimental studies argue, metaphor comprehension is context dependent. Since context includes not only the explicit physical and linguistic environment of the discourse but also its implicit cognitive environment, i.e. the beliefs and intentions of the participants, we expect that the comprehender's ability to infer the speaker's beliefs and intentions will have an effect on their interpretation of context-dependent verbal and visual stimuli. In fact, the use of 
metaphors in a communicative situation itself may be regarded as an expression of some intention. In a nutshell, we can say that the recognition of intention is relevant from all of the introduced theoretical perspectives: it relates to context in CTM, a requisite ability to understand relevant meaning in RT and an inducer of categorization in ST. For this reason, we have designed a test to quantify intention attribution ability in preschoolers. Ten short animated scenes were created in each of which two participants interact in some way. One participant (the intender) signals an intention for which the other's (the interpreter's) cooperation or help is needed. The scenes are entirely non-verbal; the intention is signalled by pointing or nodding at the object with which the interpreter's help is needed. After watching a scene, the children are shown four pictures depicting four different completions of the scene: 1) the realization of the intender's intention, 2) the intender performing an irrelevant action, 3) the interpreter performing an irrelevant action, and 4) the two participants performing an irrelevant action together. The child's task is to choose the scene completion matching the intentions of the intender.

\subsection{Short-term memory}

As discussed in section 3, we may assume that in order to interpret a metaphorical expression, the processing and organization of the target utterance, its context and/or the communicator's intention requires short-term memory. If this is the case, measures of short-term memory may help us understand the process of development. To eliminate the influence of linguistic skills on memory test performance, we choose a spatial memory test: the Corsi test (Corsi 1972). It is a non-verbal short-term memory test where the experimenter places a structure of nine randomly scattered blocks in front of the participant. The experimenter taps a sequence of blocks, one after another, and the participant's task is to repeat the sequence in exactly the same order. For young children, the sequence includes just two blocks at the first level. If the child successfully repeats the sequence, the number of blocks in the sequence increases by one.

\subsection{Non-verbal intelligence}

Non-verbal intelligence is measured by a Matrix Reasoning subtest and the Picture Concepts subtest of the Hungarian adaptation of WPPSI-IV (Kô et al. 2015). In the Matrix Reasoning task, children have to choose the correct image from four (17 trials) or five (9 trials) to complete a $2 \times 2$ matrix in a logical way. Some of the trials include items of general world knowledge (e.g. a dog, its house, and a bird are shown in the matrix, and there is a nest among the possible answers), while others demonstrate abstract entities (e.g. geometric figures of different shapes and colours). In the Picture Concepts subtest, children are shown a set of 
pictures depicting objects and have to choose two of the pictures that are in some conceptual relation with each other (e.g. choose two from the list consisting of a teddy bear, a toaster, a carnation, and a daffodil).

\subsection{Shifting and inhibition}

The children's cognitive control abilities, specifically their ability to shift between rules, i.e. inhibit the irrelevant rule and apply the relevant one, is measured using the Dimensional Change Card Sort task, which is a widely used, standardized test of cognitive control in preschool children (Müller et al. 2008, Zelazo 2006). The dimensional change card sort task (hereafter: DCCS) requires children to sort picture cards along one perceptual dimension and then, following a change in the rule, sort them along a different dimension. In order for the child to complete the task successfully, he or she must inhibit the first rule after the change and apply the second rule.

In the original version of the DCCS (Zelazo 2006), children are shown two target cards, a blue rabbit and a red boat, each of which is attached to a sorting tray. There are 18 additional cards, which are to be sorted: half of them depict a blue boat and the other half a red rabbit. Three of the blue boat cards and three of the red rabbit cards have a black frame around them. First, the children are instructed to sort the cards according to their colour: red rabbits should thus go in the tray with the red boat and blue boats should go in the tray with the blue rabbit. The children are then given one borderless sorting card at a time, and the rule is repeated at the beginning of each trial. After six trials, a new rule is introduced. The new rule is that the cards should be sorted by shape: red rabbits should go in the tray with the blue rabbit and blue boats should go in the tray with the red boat. Again, six cards need to be sorted. If a child can successfully switch to the new rule, the experiment moves on to the next level. Now the rule is that if the card has a border around it, it should be sorted by colour, but if it does not have a border around it, it should be sorted by shape. There are twelve trials at this level: three of each of the four combinations (borderless blue boat, borderless red rabbit, bordered blue boat and bordered red rabbit). It is important to note that the rule is repeated every time a card is given to the child to sort, i.e. the performance is not dependent on memory.

\subsection{Verbal intelligence}

As was discussed in section 3, core language ability also appears to be a component skill in metaphor comprehension. Verbal intelligence is measured by a subtest of the Hungarian adaptation of WPPSI-IV (Kő et al. 2015): Receptive Vocabulary. The Receptive Vocabulary task contains 31 words and phrases of increasing 
difficulty that children have to recognize by choosing the picture representing its meaning from a set of four pictures.

\section{Conclusions}

This paper is concerned with the heterogeneity of theoretical and experimental approaches to metaphor. We briefly reviewed the main principles of four theoretical models (Conceptual Metaphor Theory, Relevance Theory, Similarity Theory, and the Optimal Innovation Hypothesis) and discussed some methodological issues following from them. Next, we looked at some crucial questions in recent experimental studies on metaphor comprehension, explored possible theoretical concerns underlying each research design, and pointed out that some of the results of these works may be construed as experimental feedback on different theoretical claims. Finally, we presented our own research design based on the various features and results of previous studies.

Our main conclusion is that whether we approach the nature, comprehension, and use of metaphor from the perspective of language as an abstract system or from the point of view of the language user as a psychological phenomenon differing theoretical standpoints will point to different factors being involved in metaphor processing and will lead to divergent empirical predictions. Due to the diversity of linguistic approaches, an explorative research design may thus need to deal with some apparent contradictions (e.g. the question of age in metaphor comprehension depends on how metaphor is defined and what data collection methods are applied - see 3.4). Similarly, the theoretical heterogeneity evident in psychological theories: the variety of approaches to short-term memory, cognitive control, and verbal and nonverbal intelligence may also influence the interpretation of the results. It follows that since the chosen linguistic theory of metaphor is only one component in an experimental design the compatibility between metaphor theories and psychological models should be an issue to be reflected on and thematized when planning an experimental study. For this reason, in the current paper, we attempted to synthesize the main conclusions of a number of widely cited studies and designed a cluster of experiments in the light of these findings. Although the main experiment of the cluster is based on the Optimal Innovation Hypothesis, it is not primarily a test of a single theory: it is rather an attempt at bringing different linguistic and psychological theoretical approaches together in an empirical study of the development of metaphor comprehension. In conclusion, we believe that there is a wide range of questions in metaphor comprehension research uncovered by both theoretical and experimental studies, and, while some of the discrepancies encountered in empirical studies may be rooted in issues of methodology, they may also reflect theoretical considerations. 


\section{References}

Beaty, Roger E.-Paul J. Silvia. 2013. Metaphorically speaking: Cognitive abilities and the production of figurative language. Memory and Cognition 41(2): 255267.

Camp, Elisabeth. 2009. Two varieties of literary imagination: Metaphor, fiction, and thought experiments. Midwest Studies in Philosophy 33: 107-130.

Carriedo, Nuria Antonio Corral-Pedro R. Montoro-Laura Herrero-Patricia Ballestrino-Iraia Sebastián. 2016. The development of metaphor comprehension and its relationship with relational verbal reasoning and executive function. PLOS ONE 11(3): e0150289. DOI: 10.1371/journal. pone.0150289 (Last accessed: 17 June 2019).

Corsi, Philip Michael. 1972. Human Memory and the Medial Temporal Region of the Brain. Doctoral Thesis at McGill University (Canada).

http://digitool.library.mcgill.ca/R/?func=dbin-jump-full\&object_id=93903\& local_base=GEN01-MCG02 (Last accessed: 17 June 2019).

Deckert, Matthias-Michaela Schmoeger-Ines Schaunig-Busch-Ulrike Willinger. 2018. Metaphor processing in middle childhood and at the transition to early adolescence: The role of chronological age, mental age, and verbal intelligence. Journal of Child Language 18: 1-34.

Gardner, Howard-Ellen Winner. 1978. The development of metaphoric competence: Implications for humanistic disciplines. Critical Inquiry 5(1): 123-141.

Gardner, Howard-Mary Kircher-Ellen Winner-David Perkins. 1975. Children's metaphoric productions and preferences. Journal of Child Language 2: 125-141.

Gibbs, Raymond W, Jr.-Teenie Matlock. 2008. Metaphor, imagination, and simulation: Psycholinguistic evidence. In Raymond W. Gibbs, Jr. 2008 (ed.), The Cambridge Handbook of Metaphor and Thought, 161-176. New York, NY, US: Cambridge University Press. http://dx.doi.org/10.1017/ CBO9780511816802.011 (Last accessed: 17 June 2019).

Giora, Rachel. 1997. Understanding figurative and literal language: The graded salience hypothesis. Cognitive Linguistics 8(3): 183-206.

- 2002. Optimal innovation and pleasure. In Oliviero Stock, Carlo Strapparva, Anton Nijholt (eds), Processing of The April Fools' Day Workshop on Computational Humour, April 2002, 11-28. Trento, Italy: ITC-itst.

Giora, Rachel-Ofer Fein-Ann Kronrod-Idit Elnatan-Noa Shuval-Adi Zur. 2004. Weapons of mass distraction: Optimal innovation and pleasure ratings. Metaphor and Symbol 19(2): 115-141.

Kalandadze, Tamar-Courtenay Norbury-Terje Nærland-Kari-Anne B. Næss. 2018. Figurative language comprehension in individuals with autism spectrum disorder: A meta-analytic review. Autism 22(2): 99-117. 
Kasirer, Anat-Nira Mashal. 2014. Verbal creativity in autism: Comprehension and generation of metaphoric language in high-functioning autism spectrum disorder and typical development. Frontiers in Human Neuroscience 8. Article 615. doi.org/10.3389/fnhum.2014.00615 (Last accessed: 17 June 2019).

Kő, Natasa-Andrea Mészáros-Sándor Rózsa-Csilla Szabó-Renálta Mlinkó. 2015. WPPSI-IV Wechsler Preschool and Primary Scale of Intelligence - Fourth edition, technical and interpretative manual; Hungarian adaptation. Budapest: OS Hungary Tesztfejlesztő.

Kövecses, Zoltán. 2010 [2002]. Metaphor. A Practical Introduction. New York: Oxford University Press.

Lakoff, George-Mark L. Johnson. 1980. Metaphors We Live By. Chicago: University of Chicago Press.

Lorusso, Maria Luisa. 2007. APL-Medea. Abilità Pragmatiche Del Linguaggio. Firenze: Giunti OS.

Mashal, Nira. 2013. The role of working memory in the comprehension of unfamiliar and familiar metaphors. Language and Cognition 5(4): 409-436.

Mashal, Nira-Anat Kasirer. 2011. Thinking maps enhance metaphoric competence in children with autism and learning disabilities. Research in Developmental Disabilities 32: 2045-2054. DOI: 10.1016/j.ridd.2011.08.012 (Last accessed: 30 June 2019).

Melogno, Sergio-Maria A. Pinto-Gloria DiFilippo. 2017. Sensory and physicopsychological metaphor comprehension in children with ASD: A preliminary study on the outcomes of a treatment. Brain Sciences 7(7): 85. DOI: 10.3390/ brainsci7070085 (Last accessed: 17 June 2019).

Melogno, Sergio-Maria A. Pinto-Margherita Orsolini. 2017. Novel metaphors comprehension in a child with high-functioning autism spectrum disorder: A study on assessment and treatment. Frontiers in Psychology 7. DOI: 10.3389/ fpsyg.2016.02004 (Last accessed: 17 June 2019).

Müller, Ulrich-Philip D. Zelazo-Leah E. Lurye-Dana P. Liebermann. 2008. The effect of labeling on preschool children's performance in the Dimensional Change Card Sort Task. Cognitive Development 23: 395-408.

Özcaliskan, Seyda. 2005. On learning to draw the distinction between physical and metaphorical motion: Is metaphor an early emerging cognitive and linguistic capacity? Journal of Child Language 32(2): 291-318.

Pinto, Maria A.-Sergio Melogno-Paolo Iliceto. 2008. TCM Junior. Test di Comprensione Delle Metafore. Scuola dell'infanzia e Scuola Primaria. Roma: Carocci Faber.

Pouscoulous, Nausicaa. 2011. Metaphor: For adults only? Belgian Journal of Linguistics 25: 64-92.

Sperber, Dan-Deirdre Wilson. 1996 [1995]. Relevance: Communication and Cognition. Oxford: Blackwell. 
Stöver, Hanna. 2010. Metaphor and Relevance Theory: A New Hybrid Model. $\mathrm{PhD}$ thesis. University of Bedfordshire. http://hdl.handle.net/10547/145619 (Last accessed: 17 June 2019).

Tendahl, Markus. 2009 [2006]. A Hybrid Theory of Metaphor. Relevance Theory and Cognitive Linguistics. Houndmills, Basingstoke: Palgrave Macmillan.

Wilson, Deirdre. 2011. Parallels and differences in the treatment of metaphor in relevance theory and cognitive linguistics. Intercultural Pragmatics 8(2): 177-196.

Winner, Ellen. 1979. New names for old things: The emergence of metaphoric language. Journal of Child Language 6: 469-491.

Zelazo, Philip D. 2006. The Dimensional Change Card Sort (DCCS): A method of assessing executive function in children. Nature Protocol 1(1): 297-301. DOI: 10.1038/nprot.2006.46 (Last accessed: 17 June 2019).

Zheng, Qin-Zhongheng Jia-Dandan Liang. 2015. Metaphor and metonymy comprehension in Chinese-speaking children with high-functioning autism. Research in Autism Spectrum Disorders 10: 51-58. 